\title{
Applied Circular Dichroism: A Facile Spectroscopic Tool for Configurational Assignment and Determination of Enantiopurity
}

\author{
Macduff O. Okuom, Raychelle Burks, Crysta Naylor, and Andrea E. Holmes \\ Department of Chemistry, Doane College, 1014 Boswell Avenue, Crete, NE 68333, USA \\ Correspondence should be addressed to Macduff O. Okuom; macduff.okuom@doane.edu
}

Received 29 October 2014; Revised 7 January 2015; Accepted 8 January 2015

Academic Editor: Juan F. Garcia-Reyes

Copyright (C) 2015 Macduff O. Okuom et al. This is an open access article distributed under the Creative Commons Attribution License, which permits unrestricted use, distribution, and reproduction in any medium, provided the original work is properly cited.

In order to determine if electronic circular dichroism (ECD) is a good tool for the qualitative evaluation of absolute configuration and enantiopurity in the absence of chiral high performance liquid chromatography (HPLC), ECD studies were performed on several prescriptions and over-the-counter drugs. Cotton effects (CE) were observed for both $\mathrm{S}$ and $\mathrm{R}$ isomers between 200 and $300 \mathrm{~nm}$. For the drugs examined in this study, the $\mathrm{S}$ isomers showed a negative $\mathrm{CE}$, while the $\mathrm{R}$ isomers displayed a positive CE. The ECD spectra of both enantiomers were nearly mirror images, with the amplitude proportional to the enantiopurity. Plotting the differential extinction coefficient $(\Delta \varepsilon)$ versus enantiopurity at the wavelength of maximum amplitude yielded linear standard curves with coefficients of determination $\left(R^{2}\right)$ greater than $97 \%$ for both isomers in all cases. As expected, Equate, Advil, and Motrin, each containing a racemic mixture of ibuprofen, yielded no chiroptical signal. ECD spectra of Suphedrine and Sudafed revealed that each of them is rich in 1S,2S-pseudoephedrine, while the analysis of Equate vapor inhaler is rich in R-methamphetamine.

\section{Introduction}

Regulations of the United States Food and Drug Administration (FDA) [1] require that the properties of stereoisomers in modern drugs be known [2,3]. Although some pharmaceuticals contain racemic mixtures, such as Advil that contains both enantiomers of ibuprofen, the emphasis on enantiopure compounds being more effective than their racemic counterparts has dominated the field of chiral separation methods $[3,4]$. In the pharmaceutical industry, determination of enantiopurity is mainly done using high performance liquid chromatography (HPLC) coupled with chiral columns [5, 6]. In addition to the determination of enantiopurity, it is important to ascertain stereochemical assignments and assess the interconversion of stereoisomers $[6,7]$. The isolation of enantiomers and the assessment of optical purity still remain a challenge $[6,8,9]$. Commonly used methods for the determination of absolute configuration include electronic chiroptical spectroscopic techniques such as optical rotatory dispersion $[10,11]$, polarimetry [12], circularly polarized luminescence [10], fluorescence-detected circular dichroism
[13], and vibrational circular dichroism (VCD) [14]. Other nonchiroptical methods include nuclear magnetic resonance (NMR), mass spectrometry [15-18], X-ray crystallography [19], and chiral HPLC [20, 21].

The main purpose of this work is to show that ECD can be used as a tool to determine enantiopurity of pharmaceuticals [22] when more sophisticated methods are not readily available, such as the ones discussed in the literature [2325]. One advantage of ECD is that it can rapidly discriminate enantiomers, as well as diastereomers, and has the ability to determine the composition of racemic mixtures [26]. ECD requires millimolar or lesser concentrations, making it wellsuited for use when the quantity of the sample is limited. We explored polarimetry, but this method is well known for high analyte concentrations and large sample volume [27, 28].

We explored the possibility of using ECD as a simple tool for semiquantitative determination of enantiopurity of alanine, thalidomide, ibuprofen, methamphetamine, and pseudoephedrine (Figure 1). Alanine was selected because both enantiomers are readily available and inexpensive. 
<smiles>COC(=O)[C@@H](C)N</smiles>

S-Alanine methyl ester hydrochloride<smiles>O=C1CCC(N2C(=O)c3ccccc3C2=O)C(=O)N1</smiles>

S-Thalidomide<smiles>CC(C)Cc1ccc(C(C)C(=O)O)cc1</smiles>

S-Ibuprofen<smiles>CN[C@@H](C)Cc1ccccc1</smiles>

S-Methamphetamine

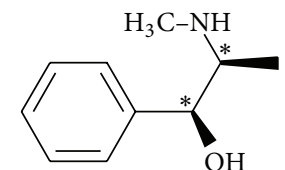

1S,2S-Pseudoephedrine<smiles>COC(=O)[C@@H](C)N</smiles>

R-Alanine methyl ester hydrochloride<smiles>O=C1CCC(N2C(=O)c3ccccc3C2=O)C(=O)N1</smiles>

R-Thalidomide<smiles>CC(C)Cc1ccc(C(C)C(=O)O)cc1</smiles>

R-Ibuprofen<smiles>CN[C@H](C)Cc1ccccc1</smiles>

R-Methamphetamine

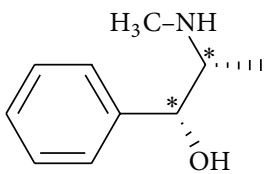

1R,2R-Pseudoephedrine

FIGURE 1: Enantiomers of alanine methyl ester hydrochloride, thalidomide, ibuprofen, methamphetamine, and pseudoephedrine. The chiral center for each enantiomer has been labeled by an asterisk $\left(^{*}\right)$.

Thalidomide was used because of its pharmaceutical relevance $[29,30]$, while ibuprofen was used because it is commercially prepared as a racemic mixture in products such as Motrin and Advil. Sudafed was used because it is commonly used as an over-the-counter (OTC) nasal decongestant and contains two chiral centers, (1S,2S)-pseudoephedrine [31]. SMethamphetamine was used for this study because it is classified in the US as a Schedule II drug. R-Methamphetamine, in contrast, is not classified as a controlled substance in the US and has the ability to constrict blood vessels. It is an ingredient in selecting OTC nasal decongestants such as Vicks vapor inhaler [32].

\section{Materials and Methods}

The following items were purchased from Sigma-Aldrich, (Milwaukee, Wisconsin): L-alanine methyl ester hydrochloride (L-alanine), D-alanine methyl ester hydrochloride (Dalanine), L-thalidomide, D-thalidomide, S-ibuprofen, deoxyephedrine (R-methamphetamine), and methamphetamine hydrochloride (S-methamphetamine). R-ibuprofen was obtained from Santa Cruz Biotechnology (Santa Cruz, California). 1S,2S- and 1R,2R-pseudoephedrine were obtained from Fisher Scientific (Pittsburgh, Pennsylvania).

Stock solutions of R- and S-alanine, R- and S-ibuprofen, and $\mathrm{R}$ - and $\mathrm{S}$-methamphetamine $(3.0 \mathrm{mM}$ in methanol) were 


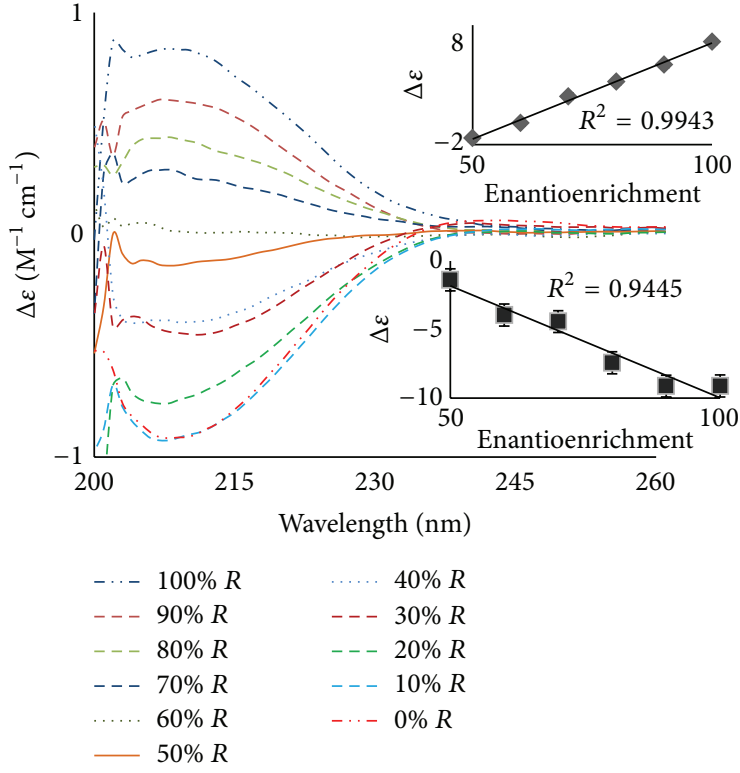

(a) Alanine CD spectra

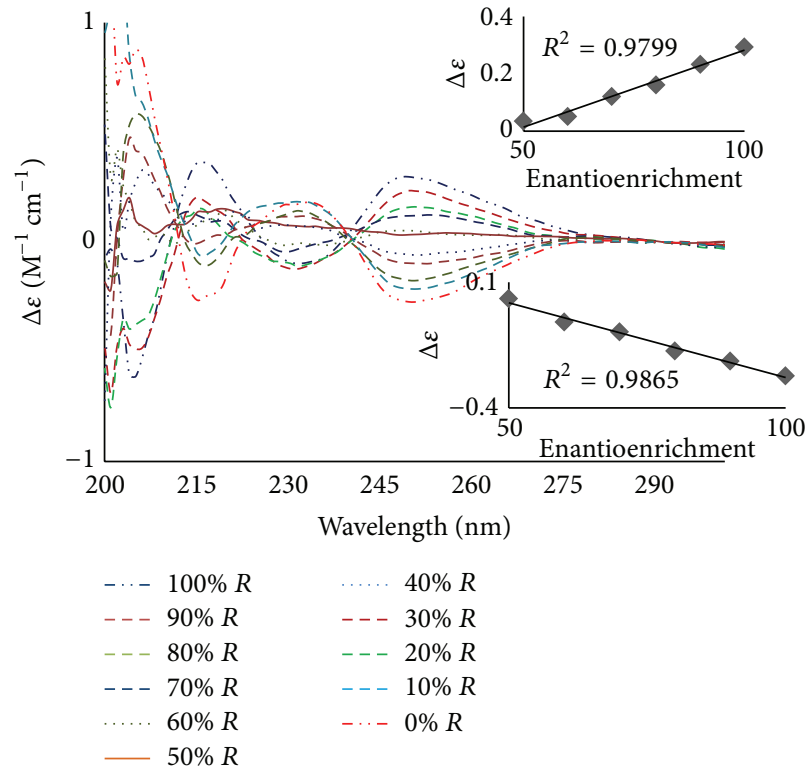

(b) Thalidomide CD spectra

FIGURE 2: CD standard curves of not-over-the-counter available substances: (a) alanine and (b) thalidomide. The spectra were measured for solutions of increasing concentration of the R-enantiomer from 0 to $100 \%$ as shown in the legend for both compounds. Insets are linear regression graphs showing between enantioenrichments versus $\Delta \varepsilon$ at the wavelength of maximum absorption. All analytes were dissolved in methanol at $0.3 \mathrm{mM}$ concentrations. All readings were determined at room temperature.

used to prepare 10 -fold dilutions of analytes. Stock solutions of R- and S-thalidomide and 1R,2R-pseudoephedrine and 1S,2S-pseudoephedrine $(0.30 \mathrm{mM}$, methanol) solutions were used to prepare 10 -fold dilutions of $(0.030 \mathrm{mM})$ of analytes.

The following were purchased from the local retail store: Equate ibuprofen, Advil, and Motrin (each with $200 \mathrm{mg}$ of ibuprofen); Sudafed and Suphedrine (each with $120 \mathrm{mg}$ of pseudoephedrine); Equate vapor inhaler (with $198 \mathrm{mg}$ of Rmethamphetamine).

Using a razor blade, the coating was removed from one pill of each of the OTC ibuprofen and then crushed using a pestle and mortar. The crushed solids were dissolved in methanol $(3.0 \mathrm{mM})$. Insoluble material was isolated and removed by centrifugation. The sponge inside the Equate vapor inhaler tube was removed and soaked in $50 \mathrm{~mL}$ of methanol for $1 \mathrm{~h}$ to extract the active ingredient, R-methamphetamine $(21.0 \mathrm{mM})$. Sample solutions of $0.3 \mathrm{mM}$ methamphetamine were made from this stock solution in methanol.

A JASCO J-815 CD spectrometer was used for sample analysis, employing a $1 \mathrm{~cm}$ quartz cuvette. Instrument conditions were as follows: room temperature setting, bandwidth of $2 \mathrm{~nm}$, response time of 1 second, standard sensitivity, wavelength range of $300-200 \mathrm{~nm}$, data pitch of $1 \mathrm{~nm}$, scanning speed of $50 \mathrm{~nm} / \mathrm{min}$, and a four-scan accumulation (averaged at end). The CD spectra of all alanine, ibuprofen, and pseudoephedrine samples were recorded in methanol at molar concentrations of $0.30 \mathrm{mM}$ at various enantiopurities (0$100 \%$ at $10 \%$ increments). The thalidomide enantiomers were investigated at $0.030 \mathrm{mM}$ concentrations under the same CD conditions. The spectra for the methamphetamine samples were normalized by subtracting a spectrum of methanol under the same conditions. Data analysis and the determination standard error were done using Microsoft Excel.

\section{Results and Discussion}

Figure 2 shows the ECD spectra for aniline and thalidomide. Figure 2(a) shows the ECD spectra for alanine. The R enantiomer has a positive $\mathrm{CE}$, while the $\mathrm{S}$ enantiomer has a negative CE centered between 200 and $240 \mathrm{~nm}$, with a maximum amplitude of $210 \mathrm{~nm}$. The $100 \%$ S-enantiomer shows an amplitude of $\Delta \varepsilon=-0.909 \mathrm{M}^{-1} \mathrm{~cm}^{-1}$, and the $100 \% \mathrm{R}$ enantiomer shows an amplitude of $\Delta \varepsilon=+0.832 \mathrm{M}^{-1} \mathrm{~cm}^{-1}$. As the enantioenrichment decreases, the amplitude decreases until it is optically inactive $\left(\Delta \varepsilon=0 \mathrm{M}^{-1} \mathrm{~cm}^{-1}\right.$ at $0 \%$ enantioenrichment). Similar results were observed for thalidomide (Figure 2(b)), ibuprofen, pseudoephedrine, and methamphetamine (Figures 3(a), 3(c), 3(e), and 4(a), resp.). Predominance by the $\mathrm{R}$ isomer gives a positive $\mathrm{CE}$, while the $\mathrm{S}$ isomer displays a negative CE. The linear relationships between the enantioenrichment and $\Delta \varepsilon$ are shown as insets and demonstrate excellent coefficients of determination (greater than $94 \%$, the standard error in the linear regression lines are shown as error bars).

In order to determine the enantiopurity of the overthe-counter (OTC) drugs, ECD spectra were obtained for $0.30 \mathrm{mM}$ methanolic solutions of Equate ibuprofen, Motrin, Advil, Sudafed, Suphedrin, and Equate vapor inhaler. No chiroptical signals were observed in the CD spectra for each of the OTC ibuprofen products (Figure 3(b)), which was expected as these substances are racemic and thus optically inactive. 


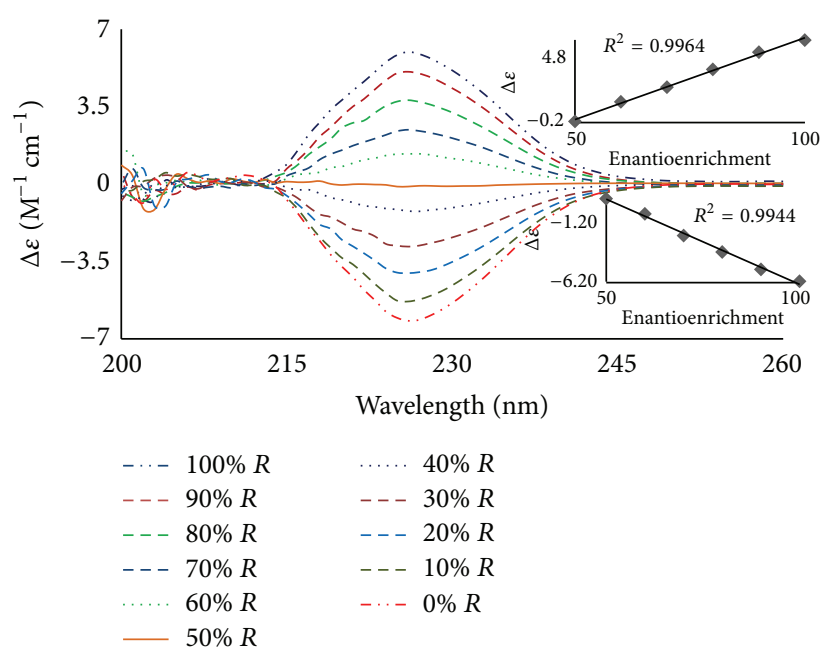

(a) Ibuprofen CD spectra

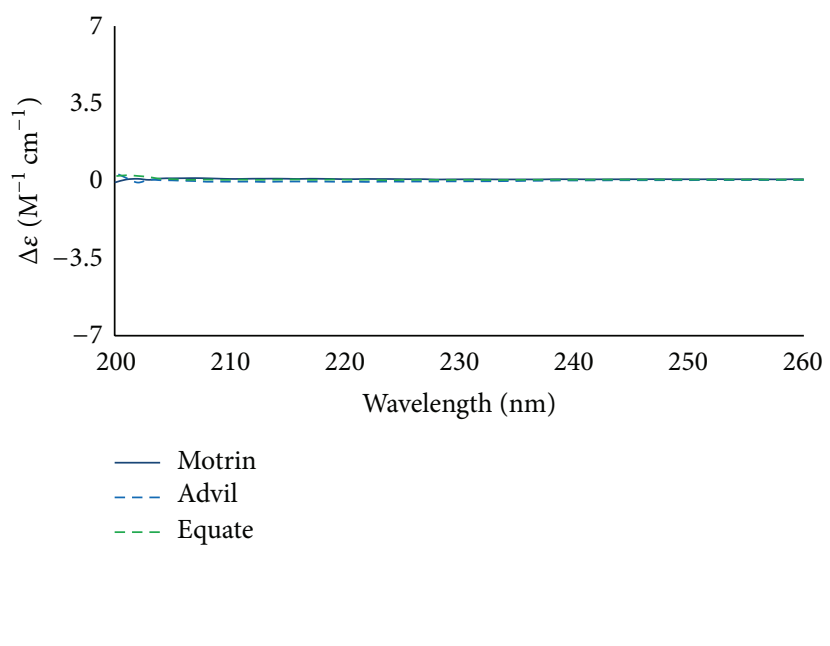

(b) CD spectra of OTC ibuprofen

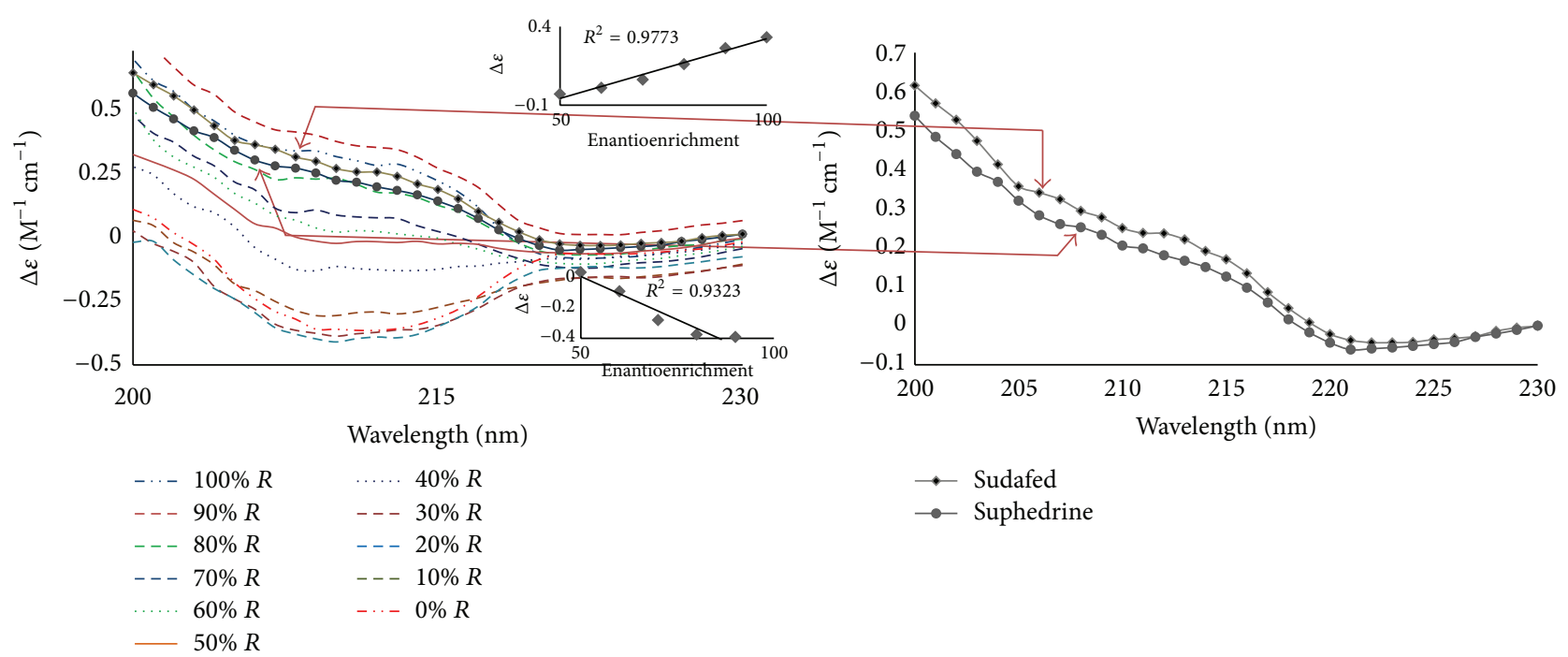

(c) Pseudoephedrine CD spectra

(d) Sudafed and Suphedrine CD spectra

FIGURE 3: CD spectra of varying enantioenrichment of the R-enantiomer (0-100\%) for OTC available substances: (a) ibuprofen, (c) pseudoephedrine, and the OTC drugs of (b) Motrin, Advil and Equate ibuprofen, (d) Sudafed and Suphedrine at $0.3 \mathrm{mM}$ concentration in methanol with a corresponding linear regression between enantioenrichment and $\Delta \varepsilon$ at the wavelength of maximum absorption shown in the inset. All readings were taken at room temperature.

Pseudoephedrine that has two chiral centers and the CD spectra were more complicated (Figure 3(c)). However, it was determined that Sudafed and Suphedrine showed an enantioenrichment of the $\mathrm{R}$ isomer of approximately $65 \%$ (Figure 3(c)), when their curves were compared to the pure pseudoephedrine curves in Figure 3(b).

The Equate vapor inhaler containing R-methamphetamine showed a $\Delta \varepsilon$ of approximately $0.135 \mathrm{M}^{-1} \mathrm{~cm}^{-1}$ at $215 \mathrm{~nm}$ (Figure 4(a)). Extrapolating this value to the standard curve of methamphetamine (Figure 4(b)), it can be estimated that the Equate vapor inhaler is at least $65 \%$ enantioenrichment of R-methamphetamine.

The enantioenrichment of $65 \%$ of Sudafed, Suphedrine, and Equate vapor inhaler seems to indicate that the OTC drugs are not quite enantiopure. While the apparent loss of enantiopurity for this result remains unclear, the possible presence of impurities leading to a complex mixture may contribute to this finding.

\section{Conclusion}

ECD spectroscopy is an easy and quick semiquantitative method for the determination of absolute configuration and enantiopurity of chiral pharmaceuticals. We demonstrated that the method does not require high sample concentrations, is user friendly, and may be used as an alternative method when chiral HPLC or HPLC coupled with ECD is not available. 


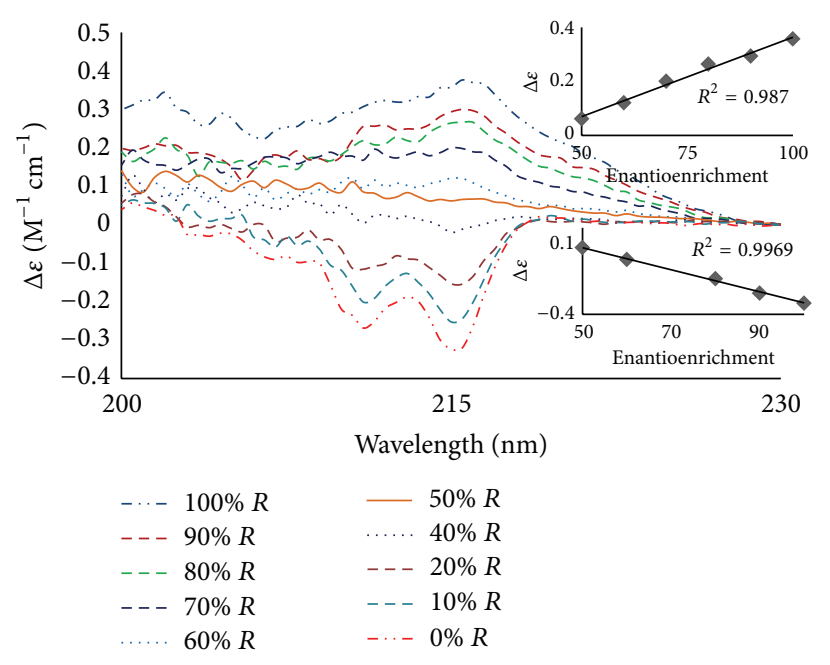

(a) Methamphetamine CD spectra

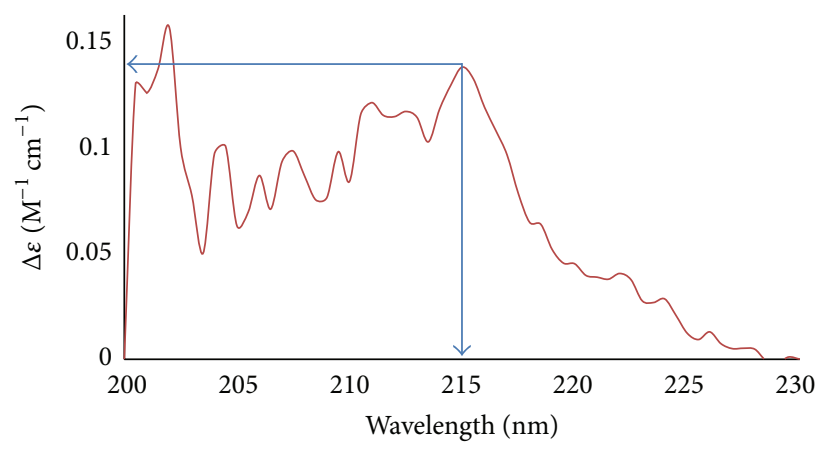

(b) Equate inhaler CD spectrum

FIGURE 4: CD spectra for (a) varying enantioenrichment of the R enantiomer (0-100\%) of $0.3 \mathrm{mM}$ methamphetamine in methanol. (b) $0.3 \mathrm{mM}$ Equate inhaler in methanol.

\section{Conflict of Interests}

The authors declare that there is no conflict of interests regarding the publication of this paper.

\section{Acknowledgments}

This research was supported by The National Science Foundation: NSF-EPSCoR-EPS-1004094 (Andrea E. Holmes, Raychelle Burks, Crysta Naylor, and Macduff O. Okuom). The authors also thank Mark Wilson and Sharmin Sikich for the valuable intellectual input.

\section{References}

[1] USFDA, “CFR—Code of Federal Regulations Title 21,” United States Food and Drug Administration, 2013.

[2] V. Buss, "Chirality, optical activity and chiropractic measuring methods," Pharmazeutische Zeitung, vol. 140, no. 4, pp. 9-22, 1995.

[3] D. H. Deutsch, "Chiral drugs: the coming revolution," Chemtech, vol. 21, pp. 157-159, 1991.

[4] S. Casal, M. B. Oliveira, and M. Ferreira, "The importance of D-amino acids in quality control of food products," Revista Portuguesa de Farmacia, vol. 49, pp. 81-86, 1999.

[5] J. Lu and A. M. Rustum, "Separation of the two enantiomers of T-3811ME by normal-phase HPLC using modified amylose as chiral stationary phase," Journal of Chromatographic Science, vol. 46, no. 6, pp. 466-471, 2008.

[6] Y. Zhang, S. Yao, H. Zeng, and H. Song, "Chiral separation of pharmaceuticals by high performance liquid chromatography," Current Pharmaceutical Analysis, vol. 6, no. 2, pp. 114-130, 2010.

[7] R. D. Shah and L. A. Nafie, "Spectroscopic methods for determining enantiomeric purity and absolute configuration in chiral pharmaceutical molecules," Current Opinion in Drug Discovery and Development, vol. 4, no. 6, pp. 764-775, 2001.
[8] N. Purdie, "Analytical application of circular dichroism," Progress in Analytical Spectroscopy, vol. 10, pp. 345-348, 1987.

[9] T. B. Freedman, X. Cao, R. K. Dukor, and L. A. Nafie, "Absolute configuration determination of chiral molecules in the solution state using vibrational circular dichroism," Chirality, vol. 15, no. 9, pp. 743-758, 2003.

[10] H. G. Brittain, "Applications of chiroptical spectroscopy for the characterization of pharmaceutical compounds," Journal of Pharmaceutical and Biomedical Analysis, vol. 17, no. 6-7, pp. 933940, 1998.

[11] H. E. Smith and R. Records, "Optically active amines-V: comparison of the optical rotatory dispersion and circular dichroism curves of the $\mathrm{N}$-salicylidene derivatives of some openchain $\alpha$-and $\beta$-arylalkylamines," Tetrahedron, vol. 22, no. 3, pp. 813-824, 1966.

[12] S. Ribe, R. K. Kondru, D. N. Beratan, and P. Wipf, "Optical rotation computation, total synthesis, and stereochemistry assignment of the marine natural product pitiamide A," Journal of the American Chemical Society, vol. 122, no. 19, pp. 4608-4617, 2000.

[13] T. Nehira, C. A. Parish, S. Jockusch, N. J. Turro, K. Nakanishi, and N. Berova, "Fluorescence-detected exciton-coupled circular dichroism: scope and limitation in structural studies of organic molecules," Journal of the American Chemical Society, vol. 121, no. 38, pp. 8681-8691, 1999.

[14] L. A. Nafie, M. R. Oboodi, and T. B. Freedman, "Vibrational circular dichroism in amino acids and peptides. 8. A chirality rule for methine $\mathrm{C} * \alpha-\mathrm{H}$ stretching modes," Journal of the American Chemical Society, vol. 105, no. 25, pp. 7449-7450, 1983.

[15] Y. Chen and Y. Wu, "Mass spectrometry of chiral compounds," Fenxi Huaxue, vol. 26, no. 6, pp. 737-741, 1998.

[16] P. Dallakian, J. Voss, and H. Budzikiewicz, "Assignment of the absolute configuration of the amino acids of pyoverdins by GC/MS," Chirality, vol. 11, no. 5-6, pp. 381-386, 1999.

[17] T. Schöpke, "Non-NMR methods for structure elucidation of saponins," in Saponins in Food, Feedstuffs and Medicinal Plants, vol. 45 of Proceedings of the Phythochemical Society of Europe, pp. 95-106, Springer, Dordrecht, The Netherlands, 2000. 
[18] R. Arakawa, M. Kobayashi, T. Fukuo, and T. Shiraiwa, "Studies on the association of 2-thiazolidinecarboxylic acid and antimony potassium tartrate: chiral recognition and prediction of absolute configuration by electrospray ionization mass spectrometry," Rapid Communications in Mass Spectrometry, vol. 15, no. 9, pp. 685-689, 2001.

[19] J. M. Bijvoet, A. F. Peerdeman, and A. J. van Bommel, "Determination of the absolute configuration of optically active compounds by means of X-rays," Nature, vol. 168, no. 4268, pp. 271-272, 1951.

[20] R. M. Woods, D. C. Patel, Y. Lim et al., "Enantiomeric separation of biaryl atropisomers using cyclofructan based chiral stationary phases," Journal of Chromatography A, vol. 1357, pp. 172-181, 2014.

[21] J. Podlech, "Origin of organic molecules and biomolecular homochirality," Cellular and Molecular Life Sciences, vol. 58, no. 1, pp. 44-60, 2001.

[22] A. Balogh-Szentesi, Application of circular dichroism (CD) spectroscopy fordetermination of pharmaceutical compounds. Analysis of stereoisomers by the HPLC-CD/UV method [Ph.D. thesis], Semmelweis University, Budapest, Hungary, 2002.

[23] I. Bustos-Jaimes, W. Hummel, T. Eggert et al., "A highthroughput screening method for chiral alcohols and its application to determine enantioselectivity of lipases and esterases," ChemCatChem, vol. 1, no. 4, pp. 445-448, 2009.

[24] Y. Chen, W. L. Tang, J. Mou, and Z. Li, "High-throughput method for determining the enantioselectivity of enzymecatalyzed hydroxylations based on mass spectrometry," Angewandte Chemie International Edition, vol. 49, no. 31, pp. 52785283, 2010.

[25] N. Berova, L. D. Bari, and G. Pescitelli, "Application of electronic circular dichroism in configurational and conformational analysis of organic compounds," Chemical Society Reviews, vol. 36, no. 6, pp. 914-931, 2007.

[26] F. Zsila, "Electronic circular dichroism spectroscopy," in Pharmaceutical Sciences Encyclopedia: Drug Discovery, Development, and Manufacturing, John Wiley \& Sons, Hoboken, NJ, USA, 2010.

[27] R. Mehvar, F. Jamali, and F. M. Pasutto, "Liquid-chromatographic assay of ibuprofen enantiomers in plasma," Clinical Chemistry, vol. 34, no. 3, pp. 493-496, 1988.

[28] UCLA, "Polarimetry," University of California, Los Angeles, Calif, USA, 2011, http://www.chem.ucla.edu/ bacher/General/ 30BL/tips/Polarimetry.html.

[29] W. G. Mcbride, "Thalidomide and congenital abnormalities," The Lancet, vol. 2, p. 1358, 1961.

[30] J. H. Siamwala, V. Veeriah, M. K. Priya et al., "Nitric oxide rescues thalidomide mediated teratogenicity," Scientific Reports, vol. 2, article 679, 2012.

[31] P. N. Patil, A. Tye, and J. B. LaPidus, "A pharmacological study of the ephedrine isomers," The Journal of Pharmacology and Experimental Therapeutics, vol. 148, pp. 158-168, 1965.

[32] W. P. Melega, A. K. Cho, D. Schmitz, R. Kuczenski, and D. S. Segal, "l-methamphetamine pharmacokinetics and pharmacodynamics for assessment of in vivo deprenyl-derived 1methamphetamine," The Journal of Pharmacology and Experimental Therapeutics, vol. 288, no. 2, pp. 752-758, 1999. 

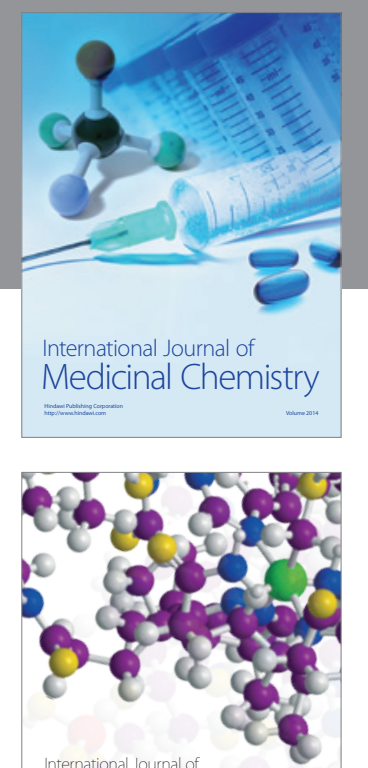

\section{Carbohydrate} Chemistry

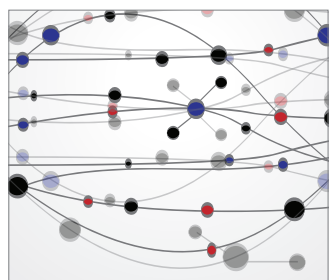

The Scientific World Journal
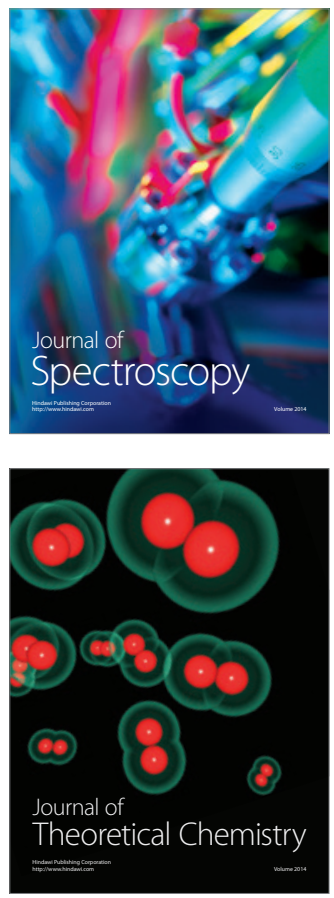
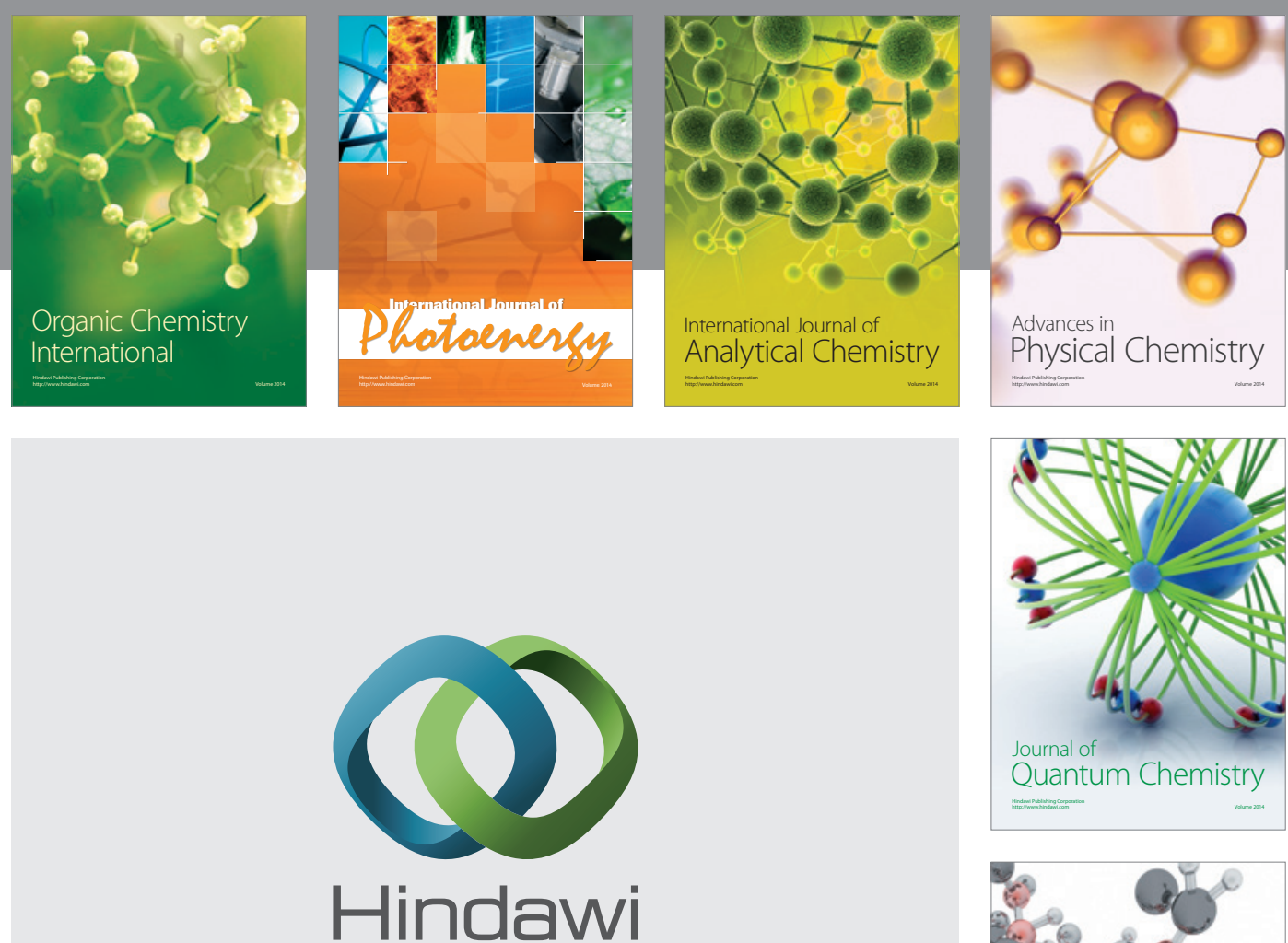

Submit your manuscripts at

http://www.hindawi.com

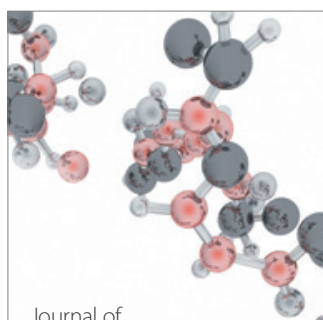

Analytical Methods

in Chemistry

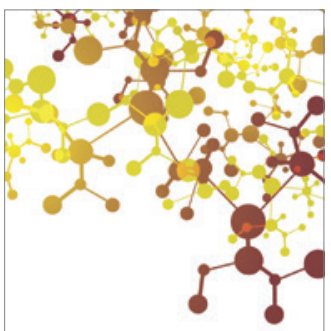

Journal of

Applied Chemistry

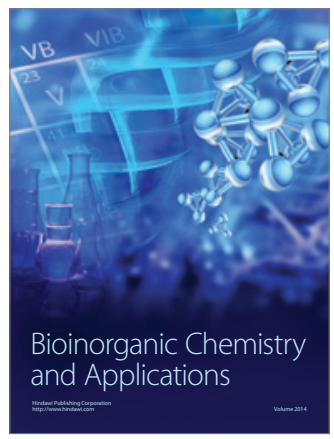

Inorganic Chemistry
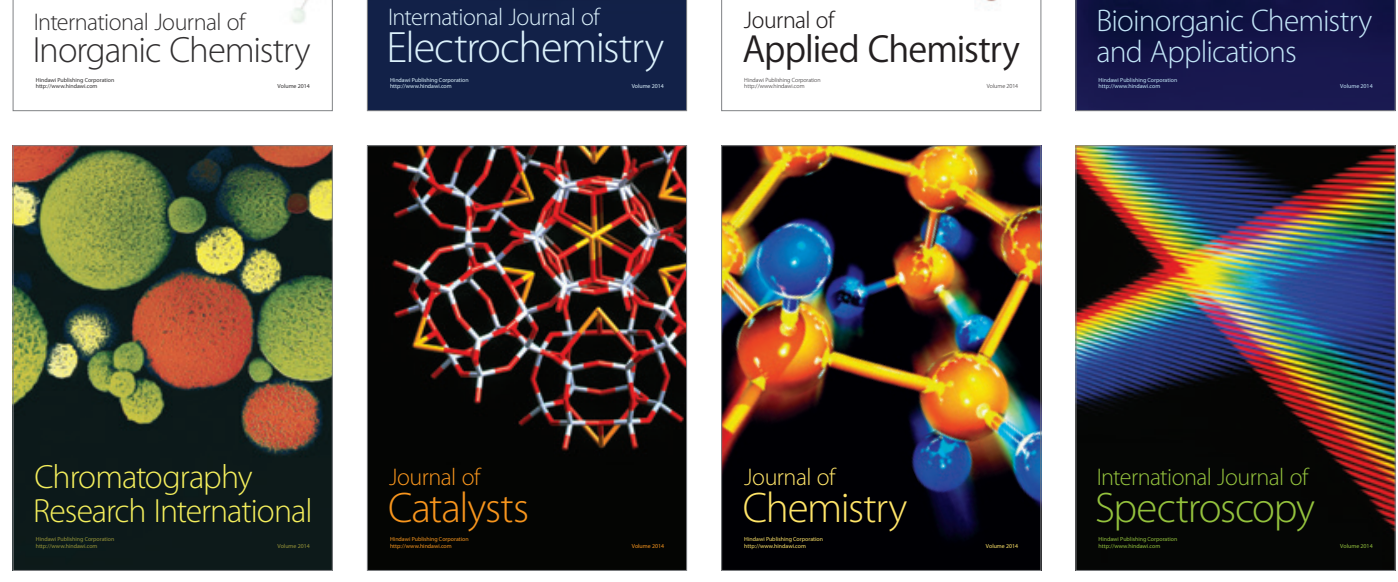